\title{
Phase-Field Approximation of Functionals Defined on Piecewise-Rigid Maps
}

\author{
Marco Cicalese $^{1}$ (D) Matteo Focardi $^{2} \cdot$ Caterina Ida Zeppieri $^{3}$
}

Received: 4 April 2021 / Accepted: 23 June 2021 / Published online: 22 July 2021

(C) The Author(s) 2021

\begin{abstract}
We provide a variational approximation of Ambrosio-Tortorelli type for brittle fracture energies of piecewise-rigid solids. Our result covers both the case of geometrically nonlinear elasticity and that of linearised elasticity.

Keywords Phase-field models · Elliptic approximation · Free-discontinuity problems $\cdot \Gamma$-convergence $\cdot$ Geometric rigidity $\cdot$ Piecewise-rigid maps $\cdot$ Linearised elasticity $\cdot$ Brittle fracture
\end{abstract}

Mathematics Subject Classification 49J45 - 49Q20 · 74B20 · 74G65

\section{Introduction}

According to the Griffith theory of crack-propagation in brittle materials (Griffith 1920), the equilibrium configuration of a fractured body is determined by balancing the reduction in bulk elastic energy $\mathcal{E}^{e}$ stored in the material with the increment in fracture energy $\mathcal{E}^{f}$ due to the formation of a new free surface. For those materials for which crack-growth can be seen as a quasi-static process, the equilibrium configurations are

Communicated by Tim Healey.

$凶 \quad$ Marco Cicalese

cicalese@ma.tum.de

Matteo Focardi

matteo.focardi@unifi.it

Caterina Ida Zeppieri

caterina.zeppieri@uni-muenster.de

1 Zentrum Mathematik - M7, Technische Universität München, Boltzmannstrasse 3, 85747

Garching, Germany

2 DiMaI “U. Dini”, Università di Firenze, V.le G.B. Morgagni 67/A, 50134 Firenze, Italia

3 Angewandte Mathematik, Universität Münster, Einsteinstr. 62, 48149 Münster, Germany 
obtained, at each time, by solving a minimisation problem involving the total free energy of the system; i.e., $\mathcal{E}:=\mathcal{E}^{e}+\mathcal{E}^{f}$.

For hyperelastic brittle materials, a prototypical elastic energy $\mathcal{E}^{e}$ is of the form

$$
\mathcal{E}^{e}(u, K)=\mu \int_{\Omega \backslash K} W(\nabla u) \mathrm{d} x,
$$

where $\Omega \subset \mathbb{R}^{3}$ is open, bounded and represents the reference configuration of a body which is fractured along a sufficiently regular closed surface $K \subset \Omega$, and $u: \Omega \backslash K \rightarrow$ $\mathbb{R}^{3}$ is the deformation map, which is smooth outside $K$. In (1.1), the constant $\mu>0$ represents the shear modulus of the material and $W: \mathbb{M}^{3 \times 3} \rightarrow[0,+\infty)$ the stored elastic energy density. In the setting of nonlinear elasticity, $W$ is assumed to be frame indifferent and to vanish only on $S O(3)$, the set of $3 \times 3$ rotation matrices; moreover, close to $S O(3)$ the function $W(\cdot)$ behaves like $\operatorname{dist}^{2}(\cdot, S O(3))$.

In a simplified isotropic setting, the fracture energy of a brittle material obeys the Griffith criterion and is proportional to the area of the crack-surface $K$, i.e.

$$
\mathcal{E}^{f}(K)=\gamma \mathcal{H}^{2}(K)
$$

where the proportionality constant $\gamma>0$ measures the fracture toughness (or fracture resistance) of the material.

Choosing $\mathcal{E}^{e}$ and $\mathcal{E}^{f}$ as in (1.1) and (1.2), respectively, the total energy $\mathcal{E}$ takes the form

$$
\mathcal{E}(u, K)=\mu \int_{\Omega \backslash K} W(\nabla u) \mathrm{d} x+\gamma \mathcal{H}^{2}(K) .
$$

The functional $\mathcal{E}$ can then be minimised by resorting to a weak formulation of the problem in De Giorgi and Ambrosio's space of special functions of bounded variation $S B V(\Omega)$ (De Giorgi and Ambrosio 1988). In this way, the pair $(u, K)$ is replaced by a single variable $u$ which can be discontinuous on a lower-dimensional set $J_{u}$, which now plays the role of the crack-surface $K$. Moreover, if $u \in S B V(\Omega)$ the distributional derivative $D u$ can be decomposed into a volume contribution $\nabla u$, which is to be interpreted as the deformation gradient outside the crack, and a surface contribution concentrated along the crack-set $J_{u}$. In the $S B V(\Omega)$-setting, the energy $\mathcal{E}$ then becomes

$$
\mathcal{F}(u)=\mu \int_{\Omega} W(\nabla u) \mathrm{d} x+\gamma \mathcal{H}^{2}\left(J_{u}\right),
$$

and its minimisation can be carried out by applying the direct methods to $\mathcal{F}$, or to its relaxed functional (cf. Ambrosio et al. 2000).

Functionals as in (1.3) are commonly referred to as free-discontinuity functionals and play a central role both in fracture mechanics (Francfort and Marigo 1998; Bourdin et al. 2000, 2008) and in computer vision (Mumford and Shah 1989) and have been extensively studied in the last decades (Ambrosio et al. 2000; Braides 1998). 
In this paper, we are interested in the case when the material parameters in (1.3) satisfy the relation $\mu / \gamma \gg 1$, or, up to a renormalisation, when $\mu \gg 1$ and $\gamma=O(1)$. This parameter-regime is typical of rigid solids; i.e., of solids which deform without storing any elastic energy. In fact, being $\mu$ large (and $\gamma=O(1)$ ), a deformation $u$ shall satisfy $W(\nabla u)=0$ which is equivalent to asking $\nabla u \in S O(3)$ almost everywhere in $\Omega$. However, since $u \in S B V(\Omega)$, the differential constraint $\nabla u \in S O$ (3) does not prevent $u$ to jump and thus fracture to occur. Hence, for $\mu \gg 1$ the energy-functional $\mathcal{F}$ models those brittle solids which exhibit a rigid behaviour in a number of subregions of $\Omega$ which are separated from one another by a discontinuity surface. Mathematically, these configurations are described by the so-called piecewise-rigid maps on $\Omega$ and are denoted by $P R(\Omega)$. Namely, $u \in P R(\Omega)$ if

$$
u(x)=\sum_{i \in \mathbb{N}}\left(\mathbb{A}_{i} x+b_{i}\right) \chi_{E_{i}}(x),
$$

where, for every $i \in \mathbb{N}, \mathbb{A}_{i} \in S O(3), b_{i} \in \mathbb{R}^{3}$, and $\left(E_{i}\right)$ is a Caccioppoli partition of $\Omega$. Then, up to a lower-order bulk-contribution, in the regime $\mu \gg 1$ the total energy $\mathcal{E}$ of a brittle rigid solid can be identified with its fracture energy; the latter coincides with the surface term in (1.3) where now the deformation-variable $u$ belongs to the space $P R(\Omega)$ (see Friedrich and Solombrino 2020).

Despite their simple analytical expression, energy functionals of type $\gamma \mathcal{H}^{n-1}\left(J_{u}\right)$ are notoriously difficult to be treated numerically, due to their explicit dependence on the discontinuity surface $J_{u}$. To develop efficient methods to compute their energy minimisers and to analyse phenomena like crack-initiation, crack-branching or arrest in nonlinearly elastic brittle materials, in the engineering community suitable "regularisations" have been recently proposed, where the surface $J_{u}$ is replaced by an additional phase-field variable $v \in[0,1]$ (see e.g., Clayton and Knap (2014); Wu et al. (2018)) and references therein). In these models the phase-filed variable $v$ interpolates between the sound state (corresponding to $v=1$ ) and the fractured state of the material (corresponding to $v=0$ ) and it is to be interpreted as a damage variable in the spirit of Pham and Marigo (2010a), Pham and Marigo (2010b), Pham et al. (2011).

It is well-known that the relationship between variational models for brittle fracture and (gradient) damage models can be made rigorous building upon the seminal approximation result of Ambrosio and Tortorelli (1990, 1992) as shown in Focardi (2001), Chambolle (2004), Bach et al. (2020), Bach et al. (2021), Bach et al., Vicente (2017), Iurlano (2014), Chambolle and Crismale (2019), just to mention a few examples. Furthermore, damage models á la Ambrosio-Tortorelli can be also used to approximate fracture models of cohesive-type as shown, e.g., in Alicandro et al. (1999), Alicandro and Focardi (2002), Conti et al. (2016), Dal Maso and Iurlano (2013), Focardi and Iurlano (2014), Iurlano (2013), Caroccia and Van Goethem (2019), Crismale (2019).

The purpose of the present paper is to establish a rigorous mathematical connection between damage models of Ambrosio-Tortorelli type and variational fracture models for brittle piecewise-rigid solids. In other words, in this work we provide an elliptic approximation of functionals of type

$$
F(u)=\gamma \mathcal{H}^{n-1}\left(J_{u}\right), \quad u \in P R(\Omega),
$$


where the constraint $u \in P R(\Omega)$ is reminiscent of the nonlinear elastic energy density $W$, which satisfy $W^{-1}(\{0\})=S O(n)$.

Namely, we show that for $(u, v) \in W^{1,2}\left(\Omega ; \mathbb{R}^{n}\right) \times W^{1,2}(\Omega), 0 \leq v \leq 1$ the family of functionals

$$
F_{\varepsilon}(u, v)=\int_{\Omega} k_{\varepsilon} v^{2} W(\nabla u) \mathrm{d} x+\frac{\gamma}{2} \int_{\Omega}\left(\frac{(v-1)^{2}}{\varepsilon}+\varepsilon|\nabla v|^{2}\right) \mathrm{d} x,
$$

converges, in the sense of De Giorgi's $\Gamma$-convergence (Dal Maso 2012; Braides 2002), to the functional (1.5), under the assumption that $k_{\varepsilon} \rightarrow+\infty$, as $\varepsilon \rightarrow 0$. As in the case of the Modica-Mortola functional (Modica 1987; Modica and Mortola 1977) and of the Ambrosio-Tortorelli approximation (Ambrosio and Tortorelli 1990, 1992), in (1.6) the singular-perturbation parameter $\varepsilon>0$ determines the thickness of the diffuse interface around the limit discontinuity surface $J_{u}$, while the diverging parameter $k_{\varepsilon}$ is proportional to the stiffness of the material, hence to the constant $\mu$ appearing in (1.3). More precisely, in Theorem 3.3 we prove that if the zero-set of the bulk energy density $W$ coincides with $S O(n)$ and for every $\mathbb{A} \in \mathbb{M}^{n \times n}$ it holds

$$
W(\mathbb{A}) \geq \alpha \operatorname{dist}^{2}(\mathbb{A}, S O(n)),
$$

for some $\alpha>0$, then the family $\left(F_{\varepsilon}\right) \Gamma$-converges to $F$, in the $L^{1}\left(\Omega ; \mathbb{R}^{n}\right) \times L^{1}(\Omega)$ topology. The proof of Theorem 3.3 takes advantage of a number of analytical tools. First, to determine the set of the limit deformations we use a piecewise-rigidity result in $S B V(\Omega)$ by Chambolle, Giacomini and Ponsiglione (Chambolle et al. 2007) (cf. Theorem 2.3). The latter is the counterpart of the Liouville rigidity theorem for deformations of brittle elastic materials and provides a characterisation of discontinuous deformations with zero elastic energy as a collection of an at most countable family of rigid motions defined on an underlying Caccioppoli partition of $\Omega$. To match the assumptions of Chambolle, Giacomini, and Ponsiglione's result we use a comparison argument with the phase-field approximation of energies in brittle fracture in [34] mentioned above. The latter takes advantage of a global argument of Ambrosio (1993) which is based on the co-area formula and it is tailor-made to gain compactness in $G S B V$. Namely, starting from a pair $\left(u_{\varepsilon}, v_{\varepsilon}\right) \subset W^{1,2}\left(\Omega, \mathbb{R}^{n}\right) \times W^{1,2}(\Omega)$ with equibounded energy $F_{\varepsilon}$ we use the co-area formula to find a suitable sublevel set of $v_{\varepsilon}$ in which $u_{\varepsilon}$ can be modified to obtain a new sequence $\left(\tilde{u}_{\varepsilon}\right) \subset G S B V\left(\Omega, \mathbb{R}^{n}\right)$ which differs from $u_{\varepsilon}$ on a set of vanishing measure and moreover satisfies

$$
\sup _{\varepsilon>0}\left(k_{\varepsilon} \int_{\Omega} \operatorname{dist}^{2}\left(\nabla \tilde{u}_{\varepsilon}, S O(n)\right) \mathrm{d} x+\mathcal{H}^{n-1}\left(J_{\tilde{u}_{\varepsilon}}\right)\right)<+\infty .
$$

The estimate above, combined with a result of Zhang which guarantees that the zeroset of the quasiconvexification of $\operatorname{dist}(\cdot, S O(n))$ coincides with $S O(n)$, proves that any $L^{1}$ limit $u$ of $\tilde{u}_{\varepsilon}$ satisfies $\nabla u \in S O(n)$ a.e. in $\Omega$. Eventually, the ChambolleGiacomini-Ponsiglione piecewise-rigidity theorem yields that $u$ is a piecewise-rigid map. The construction of Ambrosio additionally provides us with the sharp lower bound. Indeed, the perimeter of the sublevel sets of $v_{\varepsilon}$ chosen as above prove to be 
asymptotically larger than the interfacial energy-contribution of the piecewise rigid limit deformation. As in the case of the Ambrosio-Tortorelli functional, the sharp interfacial energy is defined in terms of a one-dimensional optimal profile problem. The upper bound is then proven first by resorting to a density argument and then by an explicit construction. Namely, we use the density in $P R(\Omega)$ of finite partitions subordinated to Caccioppoli sets which are polyhedral (Braides et al. 2017). Then, for these partitions, a recovery sequence matching asymptotically the sharp lower bound can be constructed by creating a layer of order $\varepsilon$ around the jump set of the target function $u$, in which the transition is one-dimensional and is obtained by a suitable scaling of the optimal profile.

As for the Ambrosio-Tortorelli approximation of the Mumford-Shah functionals (see also, e.g., Focardi 2001; Bach et al. 2020, 2021, BMZ21, Vicente 2017) also in our case the regularised bulk and surface energy in (1.6) separately converge to their sharp counterparts. Namely, in this case the bulk term in (1.6) vanishes in the limit due to the presence of the diverging parameter $k_{\varepsilon}$, that is, equivalently, limit deformations have (approximate) gradients in $S O(n)$ a.e. in $\Omega$. Similarly, the Modica-Mortola term in (1.6) approximates the limit surface energy, which in our model carries the whole energy contribution.

It is worth mentioning that the arguments in Theorem 3.3 can be extended (resorting to by-now standard modifications) to cover the case of anisotropic surface-integrals which model the presence of preferred cleavage planes in single crystals (cf. Remark 3.4).

In Theorem 4.4, we generalise the approximation result Theorem 3.3 to the case of energy densities $W$ vanishing on a compact set $\mathcal{K} \subset \mathbb{M}^{n \times n}$ for which a piecewiserigidity result analogous to the one for $S O(n)$-valued discontinuous deformations holds true. In fact in Chambolle et al. (2007), piecewise rigidity is proven, more in general, for those $\mathcal{K}$ for which a quantitative $L^{p}$-rigidity estimate holds (see Sect. 4 for more details). In this way, multiple incompatible wells can be also taken into account. From a mechanical point of view, the incompatibility describes those solids for which no fine-scale phase-mixtures are allowed in solid-solid transformations (see Müller 1999). A list of non-trivial examples of possible compact sets $\mathcal{K}$ fulfilling the assumptions of Theorem 4.4 is also included.

Finally, in Theorem 4.9 a further approximation result is provided, which covers the case of linearised elasticity.

\section{Notation and Preliminaries}

\subsection{Notation}

In what follows $\Omega \subset \mathbb{R}^{n}$ denotes a bounded domain (i.e., an open and connected set) with Lipschitz boundary. We use a standard notation for Lebesgue and Sobolev spaces, and for the Hausdorff measure. The Euclidean scalar product in $\mathbb{R}^{n}$ is denoted by $\langle\cdot, \cdot\rangle$.

We refer the reader to the book (Ambrosio et al. 2000) for a comprehensive introduction to the theory of functions of bounded variation and of (generalised) special 
functions of bounded variation $(G) S B V(\Omega)$ and to Dal Maso (2013) for the definition and main properties of generalised special functions of bounded deformation $\operatorname{GSBD}(\Omega)$. In any of these cases, we shall deal with the proper subspaces of these functional spaces in $L^{1}\left(\Omega, \mathbb{R}^{n}\right)$.

\subsection{Caccioppoli-Affine Functions}

We recall here the definition of Caccioppoli-affine and piecewise-rigid function. Moreover, we also recall the piecewise-rigidity result (Chambolle et al. 2007, Theorem 1.1) in a variant which is useful for our purposes.

Definition 2.1 A map $u: \Omega \rightarrow \mathbb{R}^{n}$ is called Caccioppoli-affine if there exist matrices $\mathbb{A}_{i} \in \mathbb{M}^{n \times n}$ and vectors $b_{i} \in \mathbb{R}^{n}$ such that

$$
u(x)=\sum_{i \in \mathbb{N}}\left(\mathbb{A}_{i} x+b_{i}\right) \chi_{E_{i}}(x),
$$

with $\left(E_{i}\right)$ Caccioppoli partition of $\Omega$. In particular, if $\mathbb{A}_{i} \in S O(n)$ for every $i \in \mathbb{N}$, then $u$ as in (2.1) is called piecewise rigid. The set of piecewise-rigid functions on $\Omega$ will be denoted by $P R(\Omega)$.

The measure theoretic properties of Caccioppoli-affine functions are collected in the result below (cf. Conti et al. 2017, Theorem 2.2).

Theorem 2.2 Let $\Omega \subset \mathbb{R}^{n}$ be open, bounded, and with Lipschitz boundary. Let $u: \Omega \rightarrow \mathbb{R}^{n}$ be Caccioppoli-affine, then $u \in(G S B V(\Omega))^{n}$. Moreover,

1. $\nabla u=\mathbb{A}_{i} \mathcal{L}^{n}$-a.e. on $E_{i}$, for every $i \in \mathbb{N}$;

2. $\mathcal{H}^{n-1}\left(J_{u} \backslash \bigcup_{i \in \mathbb{N}} \partial^{*} E_{i} \cap \varnothing\right)=0$,

Below we recall a slight generalisation of the piecewise-rigidity result by Chambolle et al. (2007, Theorem 1.1) originally stated in the $S B V$-setting. The proof follows from, e.g., Friedrich (2018, Theorem 2.3).

Theorem 2.3 Let $u \in(G S B V(\Omega))^{n}$ be such that $\mathcal{H}^{n-1}\left(J_{u}\right)<+\infty$ and $\nabla u \in S O(n)$ a.e. in $\Omega$. Then, $u \in P R(\Omega)$.

\section{Setting of the Problem and Main Result}

In this section, we introduce a family of functionals of Ambrosio-Tortorelli type (cf. Ambrosio and Tortorelli 1990, 1992) and we prove that this family converges to a surface functional of perimeter type which is finite only on piecewise-rigid maps.

Let $W: \Omega \times \mathbb{M}^{n \times n} \rightarrow[0,+\infty)$ be a Borel function such that $W(x, \mathbb{A})=0$ for every $\mathbb{A} \in S O(n)$. Assume moreover that for every $x \in \Omega$ and every $\mathbb{A} \in \mathbb{M}^{n \times n}$ it holds

$$
W(x, \mathbb{A}) \geq \alpha \operatorname{dist}^{2}(\mathbb{A}, S O(n)),
$$


for some $\alpha>0$.

Let $\Phi:[0,1] \rightarrow[0,1]$ be an increasing and lower semicontinuous function such that $\Phi(0)=0, \Phi(1)=1, \Phi(t)>0$ for $t>0$; let moreover $V:[0,1] \rightarrow[0,+\infty)$ be a continuous function with $V^{-1}(\{0\})=\{1\}$.

For $\varepsilon>0$ and $\beta>0$, we introduce the auxiliary functionals $G_{\varepsilon}^{\beta}: L^{1}\left(\Omega, \mathbb{R}^{n}\right) \times$ $L^{1}(\Omega) \longrightarrow[0,+\infty]$ defined as

$$
G_{\varepsilon}^{\beta}(u, v):= \begin{cases}\int_{\Omega}\left(\beta \Phi(v) Q\left(\operatorname{dist}^{2}(\cdot, S O(n))(\nabla u)+\frac{V(v)}{\varepsilon}+\varepsilon|\nabla v|^{2}\right) \mathrm{d} x u \in W^{1,2}\left(\Omega, \mathbb{R}^{n}\right)\right. \\ +\infty & v \in W^{1,2}(\Omega), 0 \leq v \leq 1 \\ & \text { otherwise. }\end{cases}
$$

where $Q\left(\operatorname{dist}^{2}(\cdot, S O(n))(\mathbb{A})\right.$ denotes the quasi-convex envelope of $\operatorname{dist}^{2}(\cdot, S O(n))$ computed at $\mathbb{A} \in \mathbb{M}^{n \times n}$ (cf. Giusti 2003, Section 5.3). The following Theorem is a consequence of Focardi (2001, Theorem 3.1).

Theorem 3.1 The family of functionals $\left(G_{\varepsilon}^{\beta}\right)$ defined in $(3.2) \Gamma\left(L^{1}\left(\Omega, \mathbb{R}^{n}\right) \times L^{1}(\Omega)\right)$ converges to the functional $G^{\beta}: L^{1}\left(\Omega, \mathbb{R}^{n}\right) \times L^{1}(\Omega) \longrightarrow[0,+\infty]$ given by

$$
G^{\beta}(u, v):= \begin{cases}\int_{\Omega} \beta Q\left(\operatorname{dist}^{2}(\cdot, S O(n))(\nabla u) d x+2 C_{V} \mathcal{H}^{n-1}\left(J_{u}\right)\right. & u \in\left(G S B V^{2}(\Omega)\right)^{n}, \\ +\infty & v=1 \text { a.e. on } \Omega, \\ & \text { otherwise, }\end{cases}
$$

where $C_{V}:=2 \int_{0}^{1} \sqrt{V(s)} d s$ and $G S B V^{2}(\Omega):=\{u \in G S B V(\Omega): \nabla u \in$ $\left.L^{2}\left(\Omega, \mathbb{R}^{n}\right), \mathcal{H}^{n-1}\left(S_{u}\right)<+\infty\right\}$.

Proof The proof immediately follows by Focardi (2001, Theorem 3.1) also noticing that $G S B V^{2}\left(\Omega, \mathbb{R}^{n}\right)$ coincides with $\left(G S B V^{2}(\Omega)\right)^{n}$ (see Dal Maso et al. 2005, Proposition 2.3).

For $\varepsilon>0$ let $k_{\varepsilon} \rightarrow+\infty$, as $\varepsilon \rightarrow 0$. We consider the phase-field functionals $F_{\varepsilon}: L^{1}\left(\Omega, \mathbb{R}^{n}\right) \times L^{1}(\Omega) \longrightarrow[0,+\infty]$ defined as

$F_{\varepsilon}(u, v):= \begin{cases}\int_{\Omega}\left(k_{\varepsilon} \Phi(v) W(x, \nabla u)+\frac{V(v)}{\varepsilon}+\varepsilon|\nabla v|^{2}\right) \mathrm{d} x & (u, v) \in W^{1,2}\left(\Omega, \mathbb{R}^{n}\right) \times W^{1,2}(\Omega), \\ +\infty & 0 \leq v \leq 1 \text { a.e. on } \Omega, \\ & \text { otherwise. }\end{cases}$

In the following proposition, we show that the $\Gamma$-limit of $\left(F_{\varepsilon}\right)$ (if it exists) is finite only on the set of piecewise-rigid maps.

Proposition 3.2 (Domain of the $\Gamma$-limit) Let $\left(u_{\varepsilon}, v_{\varepsilon}\right) \subset W^{1,2}\left(\Omega, \mathbb{R}^{n}\right) \times W^{1,2}(\Omega)$, $0 \leq v_{\varepsilon} \leq 1$ a.e. in $\Omega$, be such that

$$
\liminf _{\varepsilon \rightarrow 0} F_{\varepsilon}\left(u_{\varepsilon}, v_{\varepsilon}\right)<+\infty \text { and } u_{\varepsilon} \rightarrow u \text { in } L^{1}\left(\Omega, \mathbb{R}^{n}\right)
$$


Then, $v_{\varepsilon} \rightarrow 1$ in $L^{1}(\Omega)$ and $u \in P R(\Omega)$.

Proof Let $\left(u_{\varepsilon}, v_{\varepsilon}\right) \subset W^{1,2}\left(\Omega, \mathbb{R}^{n}\right) \times W^{1,2}(\Omega), 0 \leq v_{\varepsilon} \leq 1$ a.e. in $\Omega$, be such that

$$
\liminf _{\varepsilon \rightarrow 0} F_{\varepsilon}\left(u_{\varepsilon}, v_{\varepsilon}\right)<+\infty \text { and } u_{\varepsilon} \rightarrow u \text { in } L^{1}\left(\Omega, \mathbb{R}^{n}\right)
$$

We first note that up to subsequences (not relabelled) we have

$$
\sup _{\varepsilon} F_{\varepsilon}\left(u_{\varepsilon}, v_{\varepsilon}\right)<+\infty
$$

from which the convergence $v_{\varepsilon} \rightarrow 1$ in $L^{1}(\Omega)$ easily follows. In fact, for $\eta>0$ we have

$$
\mathcal{L}^{n}\left(\left\{1-\eta>v_{\varepsilon}\right\}\right) \min \{V(s): s \in[0,1-\eta)\} \leq \int_{\Omega} V\left(v_{\varepsilon}\right) \mathrm{d} x \leq \varepsilon \sup _{\varepsilon} F_{\varepsilon}\left(u_{\varepsilon}, v_{\varepsilon}\right)
$$

Since $V^{-1}(\{0\})=1$, the minimum in the left-hand side of (3.6) is strictly positive. Therefore, gathering (3.6) and (3.5) implies that $v_{\varepsilon} \rightarrow 1$ in measure. The latter, together with the uniform bound satisfied by $\left(v_{\varepsilon}\right)$ immediately gives $v_{\varepsilon} \rightarrow 1$ in $L^{1}(\Omega)$.

We are then left to show that $u \in P R(\Omega)$. Since $\operatorname{dist}^{2}(\mathbb{A}, S O(n))$ $\geq Q\left(\operatorname{dist}^{2}(\cdot, S O(n))(\mathbb{A})\right.$ and $k_{\varepsilon} \rightarrow+\infty$, appealing to Theorem 3.1 , for every $\beta>0$ we have

$$
\begin{aligned}
\liminf _{\varepsilon \rightarrow 0} F_{\varepsilon}\left(u_{\varepsilon}, v_{\varepsilon}\right) & \geq \liminf _{\varepsilon \rightarrow 0} \int_{\Omega}\left(\beta \Phi(v) \operatorname{dist}^{2}(\nabla u, S O(n))+\frac{V(v)}{\varepsilon}+\varepsilon|\nabla v|^{2}\right) \mathrm{d} x \\
& \geq \liminf _{\varepsilon \rightarrow 0} G_{\varepsilon}^{\beta}\left(u_{\varepsilon}, v_{\varepsilon}\right) \geq G^{\beta}(u, v) .
\end{aligned}
$$

As a consequence, by (3.5), we obtain that $u \in\left(G S B V^{2}(\Omega)\right)^{n}$ and therefore

$$
\mathcal{H}^{n-1}\left(J_{u}\right)<+\infty
$$

Furthermore, by the arbitrariness of $\beta>0,(3.5)$, and the very definition of $G^{\beta}$, we also get

$$
Q\left(\operatorname{dist}^{2}(\cdot, S O(n))(\nabla u)=0 \text { a.e. in } \Omega\right. \text {. }
$$

Since by Zhang (1997, Theorem 1.1) (see also Zhang 1992, Theorem 1.1) it holds

$$
\left\{\mathbb{A} \in \mathbb{M}^{n \times n}: Q\left(\operatorname{dist}^{2}(\cdot, S O(n))(\mathbb{A})=0\right\}=S O(n),\right.
$$

from (3.9) we obtain that $\nabla u \in S O(n)$ a.e.in $\Omega$. Eventually, since $u$ belongs to $(G S B V(\Omega))^{n}$ and satisfies (3.9) and (3.8), by (3.10) we can invoke Theorem 2.3 to get that $u \in P R(\Omega)$ and thus the claim. 
The next theorem establishes a $\Gamma$-convergence result for the functionals $F_{\varepsilon}$.

Theorem 3.3 The family of functionals $\left(F_{\varepsilon}\right)$ defined in $(3.4) \Gamma\left(L^{1}\left(\Omega, \mathbb{R}^{n}\right) \times L^{1}(\Omega)\right)$ converges to the functional $F: L^{1}\left(\Omega, \mathbb{R}^{n}\right) \times L^{1}(\Omega) \longrightarrow[0,+\infty]$ given by

$$
F(u, v):= \begin{cases}2 C_{V} \mathcal{H}^{n-1}\left(J_{u}\right) & \text { if } u \in P R(\Omega) \text { and } v=1 \text { a.e. in } \Omega \\ +\infty & \text { otherwise, }\end{cases}
$$

where $C_{V}:=2 \int_{0}^{1} \sqrt{V(s)} d s$.

Proof We divide the proof into two steps.

Step 1: Ansatz-free lower bound.

Let $(u, v) \in L^{1}\left(\Omega, \mathbb{R}^{n}\right) \times L^{1}(\Omega)$ be arbitrary; we need to show that for all sequences $\left(u_{\varepsilon}, v_{\varepsilon}\right) \rightarrow(u, v)$ in $L^{1}\left(\Omega, \mathbb{R}^{n}\right) \times L^{1}(\Omega), 0 \leq v_{\varepsilon} \leq 1$ a.e. in $\Omega$, we have

$$
\liminf _{\varepsilon \rightarrow 0} F_{\varepsilon}\left(u_{\varepsilon}, v_{\varepsilon}\right) \geq F(u, v) .
$$

Without loss of generality, up to the extraction of a subsequence, we may assume that the liminf in (3.12) is a limit; therefore, we have

$$
\sup _{\varepsilon} F_{\varepsilon}\left(u_{\varepsilon}, v_{\varepsilon}\right)<+\infty
$$

Then Proposition 3.2 readily implies that $u \in P R(\Omega)$ and $v=1$ a.e. in $\Omega$. For such $u$ and $v$, using again the estimate (3.7) in the proof of Proposition 3.2 we obtain the lower bound inequality (3.12) by observing that $G^{\beta}(u, 1)=2 C_{V} \mathcal{H}^{n-1}\left(J_{u}\right)$ for every $\beta>0$.

Step 2: Existence of a recovery sequence. Let $(u, v) \in L^{1}\left(\Omega, \mathbb{R}^{n}\right) \times L^{1}(\Omega)$ be arbitrary, in this step we will construct a sequence $\left(u_{\varepsilon}, v_{\varepsilon}\right) \rightarrow(u, v)$ in $L^{1}\left(\Omega, \mathbb{R}^{n}\right) \times L^{1}(\Omega)$ such that

$$
\limsup _{\varepsilon \rightarrow 0} F_{\varepsilon}\left(u_{\varepsilon}, v_{\varepsilon}\right) \leq F(u, v) .
$$

We start by noticing that the inequality in (3.14) is trivial unless we additionally assume that $u \in P R(\Omega)$ and $v=1$ a.e. in $\Omega$. Therefore, in particular we can write $u$ as

$$
u(x)=\sum_{i \in \mathbb{N}}\left(\mathbb{A}_{i} x+b_{i}\right) \chi_{E_{i}}(x)
$$

where $\mathbb{A}_{i} \in S O(n), b_{i} \in \mathbb{R}^{n}$ for every $i \in \mathbb{N}$, and $\left(E_{i}\right)$ is Caccioppoli partition of $\Omega$.

By standard density and continuity arguments (cf. (Braides 2002, Remark 1.29)) we notice that it is enough to prove (3.14) in a subset $X$ of $P R(\Omega)$, which is dense in $P R(\Omega)$ in the following sense: for every $u \in P R(\Omega)$ there exists $\left(u_{j}\right) \subset X$ such that

$$
u_{j} \rightarrow u \text { in } L^{1}\left(\Omega, \mathbb{R}^{n}\right) \text { and } \mathcal{H}^{n-1}\left(J_{u_{j}}\right) \rightarrow \mathcal{H}^{n-1}\left(J_{u}\right)
$$


for $j \rightarrow+\infty$.

We now claim that $X$ is given by those $u \in P R(\Omega)$ of the form

$$
u(x)=\sum_{i=1}^{N}\left(\widehat{\mathbb{A}}_{i} x+\hat{b}_{i}\right) \chi_{\hat{E}_{i}}(x)
$$

where $\widehat{\mathbb{A}}_{i} \in S O(n), \hat{b}_{i} \in \mathbb{R}^{n}$, and, for every $i=1, \ldots, N, \widehat{E}_{i}$ is a polyhedral set in $\Omega$. We recall that a set $\widehat{E} \subset \Omega$ is called polyhedral if there exist a finite number of $(n-1)$-dimensional simplexes $M_{1}, \ldots, M_{N} \subset \mathbb{R}^{n}$, such that $\partial \widehat{E} \cap \Omega$ coincides with $\Omega \cap \cup_{j=1}^{N} M_{j}$ up to a $\mathcal{H}^{n-1}$-null set.

Indeed given $u$ as in (3.15) the sequence $\left(u_{N}\right)$ defined as

$$
u_{N}(x)=\sum_{i=1}^{N-1}\left(\mathbb{A}_{i} x+b_{i}\right) \chi_{E_{i}}(x)+\left(\mathbb{A}_{1} x+b_{1}\right) \chi_{\Omega \backslash \bigcup_{i}^{N-1} E_{i}},
$$

clearly satisfies $u_{N} \rightarrow u$ in $L^{1}\left(\Omega, \mathbb{R}^{n}\right)$, as $N \rightarrow+\infty$. Moreover, by lower semicontinuity we have that $\mathcal{H}^{n-1}\left(J_{u}\right) \leq \liminf { }_{N} \mathcal{H}^{n-1}\left(J_{u_{N}}\right)$, hence since $\mathcal{H}^{n-1}\left(J_{u_{N}}\right) \leq$ $\mathcal{H}^{n-1}\left(J_{u}\right)$ for every $N \in \mathbb{N}$, we obtain

$$
\mathcal{H}^{n-1}\left(J_{u_{N}}\right) \rightarrow \mathcal{H}^{n-1}\left(J_{u}\right)
$$

as $N \rightarrow+\infty$.

Further, given the finite partition of $\Omega$ into sets of finite perimeter $E_{1}^{\prime}, \ldots, E_{N}^{\prime}$, with $E_{i}^{\prime}:=E_{i}$ for $i=1, \ldots, N-1$ and $E_{N}^{\prime}:=\Omega \backslash \bigcup_{i}^{N-1} E_{i}$, we can invoke (Braides et al. 2017, Corollary 2.5) to deduce the existence of a partition of $\Omega$ into polyhedral sets $\widehat{E}_{1}^{j}, \ldots, \widehat{E}_{N}^{j}$ such that, for every $i=1, \ldots, N$,

$$
\mathcal{L}^{n}\left(\widehat{E}_{i}^{j} \triangle E_{i}^{\prime}\right) \rightarrow 0 \text { and } \mathcal{H}^{n-1}\left(\partial^{*} \widehat{E}_{i}^{j}\right) \rightarrow \mathcal{H}^{n-1}\left(\partial^{*} E_{i}^{\prime}\right)
$$

as $j \rightarrow+\infty$. Eventually, the desired sequence $\left(u_{j}\right)$ satisfying (3.16)-(3.17) can be obtained by a standard diagonal argument.

We now construct a recovery sequence $\left(u_{\varepsilon}, v_{\varepsilon}\right) \subset W^{1,2}\left(\Omega, \mathbb{R}^{n}\right) \times W^{1,2}(\Omega)$ for $F_{\varepsilon}$ when $u$ is as in (3.17). Therefore, we have that, in particular, up to a set of zero $\mathcal{H}^{n-1}$-measure

$$
J_{u}=\bigcup_{i=1}^{M} S_{i} \cap \Omega
$$

where $S_{1}, \ldots, S_{M} \subset \mathbb{R}^{n}$ are a finite number of $(n-1)$-dimensional simplexes.

For every $i \in\{1, \ldots, M\}$, we denote with $\Pi_{i}$ the $(n-1)$-dimensional hyperplane containing the simplex $S_{i}$; we have that $\Pi_{i} \neq \Pi_{\ell}$, for $i \neq \ell$. 
We start by constructing $v_{\varepsilon}$. To this end, we recall that

$$
C_{V}=2 \int_{0}^{1} \sqrt{V(s)}=\inf _{T>0} \min \left\{\int_{0}^{T}\left(V(w)+\left|w^{\prime}\right|^{2}\right) \mathrm{d} t: w \in \mathcal{A}(0, T)\right\}
$$

where

$$
\mathcal{A}(0, T):=\left\{w \in W^{1, \infty}(0, T): 0 \leq w \leq 1, w(0)=0, w(T)=1\right\}
$$

(see e.g. Braides 2002, Remark 6.1). Hence, for every fixed $\eta>0$ there exists $T_{\eta}>0$ and $w_{\eta} \in W^{1, \infty}\left(0, T_{\eta}\right)$ with $0 \leq w_{\eta} \leq 1, w_{\eta}(0)=0$ and $w_{\eta}\left(T_{\eta}\right)=1$ such that

$$
\int_{0}^{T_{\eta}}\left(V\left(w_{\eta}\right)+\left|w_{\eta}^{\prime}\right|^{2}\right) \mathrm{d} t \leq C_{V}+\eta
$$

Let now $0<\xi_{\varepsilon} \ll \varepsilon$ and define the function $h_{\varepsilon}:[0,+\infty) \rightarrow[0,1]$ as

$$
h_{\varepsilon}(t):= \begin{cases}0 & \text { if } 0 \leq t \leq \xi_{\varepsilon} \\ w_{\eta}\left(\frac{t-\xi_{\varepsilon}}{\varepsilon}\right) & \text { if } \xi_{\varepsilon} \leq t \leq \xi_{\varepsilon}+\varepsilon T_{\eta} \\ 1 & \text { if } t \geq \xi_{\varepsilon}+\varepsilon T_{\eta}\end{cases}
$$

Let $\pi_{i}: \mathbb{R}^{n} \rightarrow \Pi_{i}$ denote the orthogonal projection onto $\Pi_{i}$ and set $d_{i}(x):=$ $\operatorname{dist}\left(x, \Pi_{i}\right)$, we notice that

$$
\nabla d_{i}(x)=\frac{x-\pi_{i}(x)}{\left|x-\pi_{i}(x)\right|}
$$

for every $x \in \mathbb{R}^{n} \backslash \Pi_{i}$.

Moreover, for every $\delta>0$ we define

$$
S_{i}^{\delta}:=\left\{y \in \Pi_{i}: \operatorname{dist}\left(y, S_{i}\right) \leq \delta\right\}
$$

Now let $\gamma_{\varepsilon}^{i}$ be a cut-off function between $S_{i}^{\varepsilon}$ and $S_{i}^{2 \varepsilon}$; i.e., $\gamma_{\varepsilon}^{i} \in C_{0}^{\infty}\left(S_{i}^{2 \varepsilon}\right), 0 \leq \gamma_{\varepsilon}^{i} \leq 1$, $\gamma_{\varepsilon}^{i} \equiv 1$ in $S_{i}^{\varepsilon}$, and $\left|\nabla \gamma_{\varepsilon}^{i}\right| \leq c / \varepsilon$ in $\Pi_{i}$, for some $c>0$. For every $i \in\{1, \ldots, M\}$ set

$$
v_{\varepsilon}^{i}(x):=\gamma_{\varepsilon}^{i}\left(\pi_{i}(x)\right) h_{\varepsilon}\left(d_{i}(x)\right)+1-\gamma_{\varepsilon}^{i}\left(\pi_{i}(x)\right)
$$

From the very definition of $v_{\varepsilon}^{i}$, we have that $0 \leq v_{\varepsilon}^{i} \leq 1$ and $v_{\varepsilon}^{i} \in W^{1, \infty}\left(\mathbb{R}^{n}\right)$. Moreover, by using the following facts: $\left|\nabla \gamma_{\varepsilon}^{i}\right| \leq c / \varepsilon, \pi_{i}$ is Lipschitz with constant 1 , and $\left|\nabla d_{i}\right|=1$, we also get

$$
\left\|\nabla v_{\varepsilon}^{i}\right\|_{L^{\infty}\left(\mathbb{R}^{n}\right)} \leq \frac{c}{\varepsilon}
$$


Additionally, by definition, $v_{\varepsilon}^{i} \rightarrow 1$ in $L_{\mathrm{loc}}^{1}\left(\mathbb{R}^{n}\right)$, and

$$
v_{\varepsilon}^{i} \equiv 0 \text { in } A_{i}^{\varepsilon} \text { and } v_{\varepsilon}^{i} \equiv 1 \text { in } \mathbb{R}^{n} \backslash B_{i}^{\varepsilon},
$$

where

$$
A_{i}^{\varepsilon}:=\left\{x \in \mathbb{R}^{n}: \pi_{i}(x) \in S_{i}^{\varepsilon} \text { and } d_{i}(x) \leq \xi_{\varepsilon}\right\}
$$

and

$$
B_{i}^{\varepsilon}:=\left\{x \in \mathbb{R}^{n}: \pi_{i}(x) \in S_{i}^{2 \varepsilon} \text { and } d_{i}(x) \leq \xi_{\varepsilon}+\varepsilon T_{\eta}\right\} .
$$

Therefore, in view of (3.24) we have

$$
\int_{B_{i}^{\varepsilon}}\left(\frac{V\left(v_{\varepsilon}^{i}\right)}{\varepsilon}+\varepsilon\left|\nabla v_{\varepsilon}^{i}\right|^{2}\right) \mathrm{d} x=\frac{V(0)}{\varepsilon} \mathcal{L}^{n}\left(A_{i}^{\varepsilon}\right)+\int_{B_{i}^{\varepsilon} \backslash A_{i}^{\varepsilon}}\left(\frac{V\left(v_{\varepsilon}^{i}\right)}{\varepsilon}+\varepsilon\left|\nabla v_{\varepsilon}^{i}\right|^{2}\right) \mathrm{d} x
$$

moreover, we notice that

$$
\lim _{\varepsilon \rightarrow 0} \frac{V(0)}{\varepsilon} \mathcal{L}^{n}\left(A_{i}^{\varepsilon}\right)=2 V(0) \lim _{\varepsilon \rightarrow 0} \frac{\xi_{\varepsilon}}{\varepsilon} \mathcal{H}^{n-1}\left(S_{i}^{\varepsilon}\right)=0
$$

where to establish the last equality we have used that $\xi_{\varepsilon} \ll \varepsilon$ and $\mathcal{H}^{n-1}\left(S_{i}^{\varepsilon}\right) \rightarrow$ $\mathcal{H}^{n-1}\left(S_{i}\right)$, as $\varepsilon \rightarrow 0$. We now estimate the second term in the right-hand side of (3.25). To do so, it is convenient to write

$$
B_{i}^{\varepsilon} \backslash A_{i}^{\varepsilon}=H_{i}^{\varepsilon} \cup I_{i}^{\varepsilon}
$$

where

$$
H_{i}^{\varepsilon}:=\left\{x \in \mathbb{R}^{n}: \pi_{i}(x) \in S_{i}^{\varepsilon} \text { and } \xi_{\varepsilon} \leq d_{i}(x) \leq \xi_{\varepsilon}+\varepsilon T_{\eta}\right\}
$$

and

$$
I_{i}^{\varepsilon}:=\left\{x \in \mathbb{R}^{n}: \pi_{i}(x) \in S_{i}^{2 \varepsilon} \backslash S_{i}^{\varepsilon} \text { and } d_{i}(x) \leq \xi_{\varepsilon}+\varepsilon T_{\eta}\right\}
$$

By definition of $v_{\varepsilon}^{i}$, in the set $H_{i}^{\varepsilon}$ it holds

$$
v_{\varepsilon}^{i}(x)=w_{\eta}\left(\frac{d_{i}(x)-\xi_{\varepsilon}}{\varepsilon}\right)
$$

and thus

$$
\nabla v_{\varepsilon}^{i}(x)=\frac{1}{\varepsilon} w_{\eta}^{\prime}\left(\frac{d_{i}(x)-\xi_{\varepsilon}}{\varepsilon}\right) \nabla d_{i}(x)
$$


Therefore, since $\left|\nabla d_{i}\right|=1$ a.e., we have

$$
\begin{aligned}
& \int_{H_{i}^{\varepsilon}}\left(\frac{V\left(v_{\varepsilon}^{i}\right)}{\varepsilon}+\varepsilon\left|\nabla v_{\varepsilon}^{i}\right|^{2}\right) \mathrm{d} x \\
& =\int_{H_{i}^{\varepsilon}}\left(\frac{1}{\varepsilon} V\left(w_{\eta}\left(\frac{d_{i}(x)-\xi_{\varepsilon}}{\varepsilon}\right)\right)+\varepsilon\left|\frac{1}{\varepsilon} w_{\eta}^{\prime}\left(\frac{d_{i}(x)-\xi_{\varepsilon}}{\varepsilon}\right) \nabla d_{i}(x)\right|^{2}\right) \mathrm{d} x \\
& =2 \int_{S_{i}^{\varepsilon}} d \mathcal{H}^{n-1} \int_{\xi_{\varepsilon}}^{\xi_{\varepsilon}+\varepsilon T_{\eta}}\left(\frac{1}{\varepsilon} V\left(w_{\eta}\left(\frac{t-\xi_{\varepsilon}}{\varepsilon}\right)\right)+\frac{1}{\varepsilon}\left|w_{\eta}^{\prime}\left(\frac{t-\xi_{\varepsilon}}{\varepsilon}\right)\right|^{2}\right) \mathrm{d} t \\
& =2 \int_{S_{i}^{\varepsilon}} d \mathcal{H}^{n-1} \int_{0}^{T_{\eta}}\left(V\left(w_{\eta}(t)\right)+\left|w_{\eta}^{\prime}(t)\right|^{2}\right) \mathrm{d} t \\
& \leq 2\left(C_{V}+\eta\right) \mathcal{H}^{n-1}\left(S_{i}\right)+o(1)
\end{aligned}
$$

as $\varepsilon \rightarrow 0$, where to establish the last inequality we have used (3.20).

Furthermore, from (3.23) it is immediate to show that

$$
\lim _{\varepsilon \rightarrow 0} \int_{I_{i}^{\varepsilon}}\left(\frac{V\left(v_{\varepsilon}^{i}\right)}{\varepsilon}+\varepsilon\left|\nabla v_{\varepsilon}^{i}\right|^{2}\right) \mathrm{d} x \leq \lim _{\varepsilon \rightarrow 0} \frac{c}{\varepsilon} \varepsilon \mathcal{H}^{n-1}\left(S_{i}^{2 \varepsilon} \backslash S_{i}^{\varepsilon}\right)=0 .
$$

Eventually, gathering (3.25)-(3.30) yields

$$
\int_{B_{i}^{\varepsilon}}\left(\frac{V\left(v_{\varepsilon}^{i}\right)}{\varepsilon}+\varepsilon\left|\nabla v_{\varepsilon}^{i}\right|^{2}\right) \mathrm{d} x \leq 2\left(C_{V}+\eta\right) \mathcal{H}^{n-1}\left(S_{i}\right)+o(1),
$$

as $\varepsilon \rightarrow 0$.

Now the idea is to combine together the sequences $\left(v_{\varepsilon}^{i}\right)$ in order to define a new sequence $\left(v_{\varepsilon}\right)$ which belongs to $W^{1,2}(\Omega)$ and in every $B_{i}^{\varepsilon}$ coincides with $\left(v_{\varepsilon}^{i}\right)$, up to a set where the surface energy is negligible. Moreover, the sequence $\left(v_{\varepsilon}\right)$ shall satisfy: $v_{\varepsilon} \rightarrow 1$ in $L^{1}(\Omega)$ and

$$
\limsup _{\varepsilon \rightarrow 0} \int_{\Omega}\left(\frac{V\left(v_{\varepsilon}^{i}\right)}{\varepsilon}+\varepsilon\left|\nabla v_{\varepsilon}^{i}\right|^{2}\right) \mathrm{d} x \leq 2\left(C_{V}+\eta\right) \mathcal{H}^{n-1}\left(J_{u}\right),
$$

where $u$ is as in (3.17).

To this end, we define

$$
v_{\varepsilon}:=\min \left\{v_{\varepsilon}^{1}, \ldots, v_{\varepsilon}^{M}\right\}
$$

clearly, $0 \leq v_{\varepsilon} \leq 1,\left(v_{\varepsilon}\right) \subset W^{1,2}(\Omega)$, and $v_{\varepsilon} \rightarrow 1$ in $L^{1}(\Omega)$ hence, in particular, $v_{\varepsilon} \rightarrow 1$ in $L^{1}(\Omega)$. Further, setting

$$
A^{\varepsilon}:=\bigcup_{i=1}^{M} A_{i}^{\varepsilon} \text { and } B^{\varepsilon}:=\bigcup_{i=1}^{M} B_{i}^{\varepsilon}
$$


by (3.24) and (3.32) we readily deduce that

$$
v_{\varepsilon} \equiv 0 \text { in } A^{\varepsilon} \text { and } v_{\varepsilon} \equiv 1 \text { in } \mathbb{R}^{n} \backslash B^{\varepsilon} \text {. }
$$

Then, writing $\Omega=\left(\Omega \backslash B^{\varepsilon}\right) \cup\left(\Omega \cap\left(B^{\varepsilon} \backslash A^{\varepsilon}\right)\right) \cup\left(\Omega \cap A^{\varepsilon}\right)$, in view of (3.33) we get

$$
\begin{aligned}
\int_{\Omega}\left(\frac{V\left(v_{\varepsilon}^{i}\right)}{\varepsilon}+\varepsilon\left|\nabla v_{\varepsilon}^{i}\right|^{2}\right) \mathrm{d} x \leq & \int_{\Omega \cap\left(B^{\varepsilon} \backslash A^{\varepsilon}\right)}\left(\frac{V\left(v_{\varepsilon}^{i}\right)}{\varepsilon}+\varepsilon\left|\nabla v_{\varepsilon}^{i}\right|^{2}\right) \mathrm{d} x \\
& +2 V(0) \frac{\xi_{\varepsilon}}{\varepsilon} \sum_{i=1}^{M} \mathcal{H}^{n-1}\left(S_{i}^{\varepsilon}\right) .
\end{aligned}
$$

Since $\xi_{\varepsilon} \ll \varepsilon$ and

$$
\sum_{i=1}^{M} \mathcal{H}^{n-1}\left(S_{i}^{\varepsilon}\right) \rightarrow \sum_{i=1}^{M} \mathcal{H}^{n-1}\left(S_{i}\right)=\mathcal{H}^{n-1}\left(J_{u}\right)
$$

as $\varepsilon \rightarrow 0$, the second term in the right-hand side of (3.34) is negligible. Hence, to get (3.31) we are left to estimate the surface energy in $\Omega \cap\left(B^{\varepsilon} \backslash A^{\varepsilon}\right)$. We claim that

$$
\limsup _{\varepsilon \rightarrow 0} \mathcal{S}_{\varepsilon}:=\limsup _{\varepsilon \rightarrow 0} \int_{\Omega \cap\left(B^{\varepsilon} \backslash A^{\varepsilon}\right)}\left(\frac{V\left(v_{\varepsilon}\right)}{\varepsilon}+\varepsilon\left|\nabla v_{\varepsilon}\right|^{2}\right) \mathrm{d} x \leq 2\left(C_{V}+\eta\right) \mathcal{H}^{n-1}\left(J_{u}\right)
$$

We notice that

$$
B^{\varepsilon} \backslash A^{\varepsilon}=\bigcup_{i=1}^{M}\left(H_{i}^{\varepsilon} \cup I_{i}^{\varepsilon}\right) \backslash A^{\varepsilon}
$$

where the sets $H_{i}^{\varepsilon}$ and $I_{i}^{\varepsilon}$ are defined as in (3.27) and (3.28), respectively. Since

$$
\left(H_{i}^{\varepsilon} \cup I_{i}^{\varepsilon}\right) \backslash A^{\varepsilon} \subset \bigcup_{j \neq i}\left(\left(H_{i}^{\varepsilon} \cup I_{i}^{\varepsilon}\right) \cap\left(H_{j}^{\varepsilon} \cup I_{j}^{\varepsilon}\right)\right) \cup \bigcap_{j \neq i}\left(\left(H_{i}^{\varepsilon} \cup I_{i}^{\varepsilon}\right) \backslash B_{j}^{\varepsilon}\right),
$$

we have

$$
\begin{aligned}
\mathcal{S}_{\varepsilon} \leq & \sum_{i=1}^{M} \int_{\Omega \cap\left(\left(H_{i}^{\varepsilon} \cup I_{i}^{\varepsilon}\right) \backslash A^{\varepsilon}\right)}\left(\frac{V\left(v_{\varepsilon}\right)}{\varepsilon}+\varepsilon\left|\nabla v_{\varepsilon}\right|^{2}\right) \mathrm{d} x \\
\leq & \sum_{i=1}^{M} \int_{\left.\Omega \cap \bigcap_{j \neq i}\left(\left(H_{i}^{\varepsilon} \cup I_{i}^{\varepsilon}\right) \backslash B_{j}^{\varepsilon}\right)\right)}\left(\frac{V\left(v_{\varepsilon}\right)}{\varepsilon}+\varepsilon\left|\nabla v_{\varepsilon}\right|^{2}\right) \mathrm{d} x \\
& +\sum_{i=1}^{M} \int_{\Omega \cap \bigcup_{j \neq i}\left(\left(H_{i}^{\varepsilon} \cup I_{i}^{\varepsilon}\right) \cap\left(H_{j}^{\varepsilon} \cup I_{j}^{\varepsilon}\right)\right)}\left(\frac{V\left(v_{\varepsilon}\right)}{\varepsilon}+\varepsilon\left|\nabla v_{\varepsilon}\right|^{2}\right) \mathrm{d} x
\end{aligned}
$$




$$
=: \mathcal{S}_{\varepsilon}^{1}+\mathcal{S}_{\varepsilon}^{2}
$$

We now estimate the terms $\mathcal{S}_{\varepsilon}^{1}$ and $\mathcal{S}_{\varepsilon}^{2}$ separately. To this end, we start observing that

$$
\bigcap_{j \neq i}\left(\left(H_{i}^{\varepsilon} \cup I_{i}^{\varepsilon}\right) \backslash B_{j}^{\varepsilon}\right) \subset \bigcap_{j \neq i}\left\{x \in \mathbb{R}^{n}: v_{\varepsilon}^{i}(x) \leq v_{\varepsilon}^{j}(x)\right\},
$$

hence, invoking (3.29) and (3.30), we readily get

$$
\begin{aligned}
\mathcal{S}_{\varepsilon}^{1} & =\sum_{i=1}^{M} \int_{\left.\Omega \cap \bigcap_{j \neq i}\left(\left(H_{i}^{\varepsilon} \cup I_{i}^{\varepsilon}\right) \backslash B_{j}^{\varepsilon}\right)\right)}\left(\frac{V\left(v_{\varepsilon}^{i}\right)}{\varepsilon}+\varepsilon\left|\nabla v_{\varepsilon}^{i}\right|^{2}\right) \mathrm{d} x \\
& \leq\left(2 C_{V}+\eta\right) \sum_{i=1}^{M} \mathcal{H}^{n-1}\left(S_{i}\right)+o(1) \\
& =\left(2 C_{V}+\eta\right) \mathcal{H}^{n-1}\left(J_{u}\right)+o(1),
\end{aligned}
$$

as $\varepsilon \rightarrow 0$. Moreover, appealing to (3.23) easily gives

$$
\mathcal{S}_{\varepsilon}^{2} \leq \sum_{i=1}^{M} \sum_{j \neq i} \frac{c}{\varepsilon} \mathcal{L}^{n}\left(\left(H_{i}^{\varepsilon} \cup I_{i}^{\varepsilon}\right) \cap\left(H_{j}^{\varepsilon} \cup I_{j}^{\varepsilon}\right)\right) .
$$

We now claim that

$$
\lim _{\varepsilon \rightarrow 0} \frac{c}{\varepsilon} \mathcal{L}^{n}\left(\left(H_{i}^{\varepsilon} \cup I_{i}^{\varepsilon}\right) \cap\left(H_{j}^{\varepsilon} \cup I_{j}^{\varepsilon}\right)\right)=0,
$$

for every $i, j \in\{1, \ldots, M\}$. Indeed, since $\Pi_{i} \neq \Pi_{j}$ then the set $S_{i} \cap S_{j}$ is contained in an $(n-2)$-dimensional affine subspace of $\mathbb{R}^{n}$, so that by (3.27) and (3.28) we can deduce that

$$
\mathcal{L}^{n}\left(\left(H_{i}^{\varepsilon} \cup I_{i}^{\varepsilon}\right) \cap\left(H_{j}^{\varepsilon} \cup I_{j}^{\varepsilon}\right)\right) \leq c\left(\xi_{\varepsilon}+\varepsilon T_{\eta}\right)^{2}=O\left(\varepsilon^{2}\right),
$$

as $\varepsilon \rightarrow 0$, where the constant $c>0$ depends only on the angle between $\Pi_{i}$ and $\Pi_{j}$ and on $\mathcal{H}^{n-2}\left(S_{i} \cap S_{j}\right)$. Hence, (3.40) immediately yields (3.39).

Finally, gathering (3.36) and (3.37) entails (3.35), as desired.

Therefore, to conclude the proof of the upper bound we now have to exhibit a sequence $\left(u_{\varepsilon}\right) \subset W^{1,2}\left(\Omega, \mathbb{R}^{n}\right)$ such that $u_{\varepsilon} \rightarrow u$ in $L^{1}\left(\Omega, \mathbb{R}^{n}\right)$ and

$$
\limsup _{\varepsilon \rightarrow 0} \int_{\Omega} k_{\varepsilon} \Phi\left(v_{\varepsilon}\right) W\left(x, \nabla u_{\varepsilon}\right) \mathrm{d} x=0 .
$$

To this end, set

$$
\left(A^{\varepsilon}\right)^{\prime}:=\bigcup_{i=1}^{M}\left\{x \in \mathbb{R}^{n}: \pi_{i}(x) \in S_{i}^{\varepsilon / 2} \text { and } d_{i}(x) \leq \frac{\xi_{\varepsilon}}{2}\right\}
$$


let $\varphi_{\varepsilon} \in C_{0}^{\infty}\left(A^{\varepsilon}\right)$ be a cut-off function between $\left(A^{\varepsilon}\right)^{\prime}$ and $A^{\varepsilon}$, and define

$$
u_{\varepsilon}:=\left(1-\varphi_{\varepsilon}\right) u
$$

Then, clearly $\left(u_{\varepsilon}\right) \subset W^{1, \infty}\left(\Omega, \mathbb{R}^{n}\right)$, moreover $u_{\varepsilon} \rightarrow u$ in $L^{1}\left(\Omega, \mathbb{R}^{n}\right)$. Moreover, since $v_{\varepsilon} \equiv 0$ in $A^{\varepsilon}$, and $\Phi$ vanishes at zero, it holds

$$
\int_{\Omega} k_{\varepsilon} \Phi\left(v_{\varepsilon}\right) W\left(x, \nabla u_{\varepsilon}\right) \mathrm{d} x=\int_{\Omega \backslash A^{\varepsilon}} k_{\varepsilon} \Phi\left(v_{\varepsilon}\right) W(x, \nabla u) \mathrm{d} x
$$

hence using that $u \in P R(\Omega)$ together with the fact that for every $x \in \Omega$ the function $W(x, \cdot)$ vanishes in $S O(n)$ we immediately get

$$
\Phi\left(v_{\varepsilon}\right) W(x, \nabla u)=0 \text { a.e. in } \Omega \backslash A^{\varepsilon}
$$

and hence the claim.

Remark 3.4 (Approximation of inhomogeneous anisotropic perimeter functionals) Arguing as in the proof of Vicente (2017) (see also Focardi 2001, Theorem 3.1), in view of Proposition 3.2 one can establish a $\Gamma$-convergence result for functionals of the form

$F_{\varepsilon}^{\phi}(u, v):=\left\{\begin{array}{lc}\int_{\Omega}\left(k_{\varepsilon} \Phi(v) W(x, \nabla u)+\frac{V(v)}{\varepsilon}+\varepsilon \phi^{2}(x, \nabla v)\right) \mathrm{d} x(u, v) \in W^{1,2}\left(\Omega, \mathbb{R}^{n}\right) \times W^{1,2}(\Omega), \\ +\infty & 0 \leq v \leq 1 \text { a.e. on } \Omega, \\ \text { otherwise, }\end{array}\right.$

where the euclidean norm in $F_{\varepsilon}$ is now replaced by a Finsler norm $\phi$. That is, $\phi: \Omega \times$ $\mathbb{R}^{n} \rightarrow[0,+\infty)$ is a continuous function which is convex in its second variable and satisfies the two following properties:

i. for every $(x, z) \in \Omega \times \mathbb{R}^{n}$ and for every $t \in \mathbb{R}$

$$
\phi(x, t z)=|t| \phi(x, z)
$$

ii. for every $(x, z) \in \Omega \times \mathbb{R}^{n}$ there exist $0<m \leq M<+\infty$ such that

$$
m|z| \leq \phi(x, z) \leq M|z|
$$

In this case, it can be proven that the family of functionals $\left(F_{\varepsilon}^{\phi}\right) \Gamma\left(L^{1}\left(\Omega, \mathbb{R}^{n}\right) \times\right.$ $L^{1}(\Omega)$ )-converges to the following inhomogeneous and anisotropic functional $F^{\phi}: L^{1}\left(\Omega, \mathbb{R}^{n}\right) \times L^{1}(\Omega) \longrightarrow[0,+\infty]$ defined on piecewise rigid maps as:

$$
F^{\phi}(u, v):= \begin{cases}2 C_{V} \int_{J_{u}} \phi\left(x, v_{u}\right) d \mathcal{H}^{n-1} & \text { if } u \in P R(\Omega) \text { and } v=1 \text { a.e. in } \Omega \\ +\infty & \text { otherwise }\end{cases}
$$

where $v_{u}$ denotes the exterior unit normal to $J_{u}$. 


\section{Incompatible Wells and Linearised Elasticity}

In this section, we are going to address two possible extensions of Theorem 3.3. We first discuss a generalisation of Theorem 3.3 to the case where the zeros of the potential $W$ lie in a suitable nonempty compact set $\mathcal{K}$. Then, we show that our proof-strategy also applies to the case of linearised elasticity. Similarly as in Sect. 3, also in these cases the key tools for the analysis are two suitable variants of the piecewise-rigidity property stated in Theorem 2.3 (cf. Theorems 4.2 and 4.6).

\subsection{The Case of $\mathcal{K}$ Piecewise-Rigid Maps}

Let $U \subset \mathbb{R}^{n}$ be a bounded domain with Lipschitz boundary, and let $\mathcal{K} \subset \mathbb{M}^{n \times n}$ be a nonempty compact set satisfying the following $L^{p}$-quantitative rigidity estimate for some $p \in(1, n /(n-1))$ : there exists a constant $C>0$ (depending only on $p$ and $n$ ) such that for every $u \in W^{1, p}\left(U, \mathbb{R}^{n}\right)$

$$
\min _{\mathbb{A} \in \mathcal{K}}\|\nabla u-\mathbb{A}\|_{L^{p}\left(U, \mathbb{M}^{n \times n}\right)} \leq C\|\operatorname{dist}(\nabla u, \mathcal{K})\|_{L^{p}(U)}
$$

We notice that (4.1) implies the rigidity of the differential inclusion

$$
v \in W^{1, \infty}\left(U, \mathbb{R}^{n}\right) \text { and } \nabla v(x) \in \mathcal{K} \quad \text { a.e. } U,
$$

in the sense explained in Lemma 4.1. In the statement of Lemma 4.1, we use the same terminology adopted in Rindler (2018, Chapter 8) (see also Müller 1999, Section 1.4 and Kirchheim 2003).

Lemma 4.1 Let $U \subset \mathbb{R}^{n}$ be a bounded domain with Lipschitz boundary. Let $\mathcal{K} \subset$ $\mathbb{M}^{n \times n}$ be a nonempty compact set satisfying (4.1). Then, the following statements hold true:

(1) the differential inclusion (4.2) is rigid for exact solutions; i.e., the only solutions to (4.2) are affine functions;

(2) the differential inclusion (4.2) is rigid for approximate solutions; i.e., if $\operatorname{dist}\left(\nabla u_{j}, \mathcal{K}\right) \rightarrow 0$ in measure in $U, \quad\left(u_{j}\right)$ converges to $u$ weakly* in $W^{1, \infty}\left(U, \mathbb{R}^{n}\right), u_{j}=\mathbb{A} x$ on $\partial U$ for some $\mathbb{A} \in \mathbb{M}^{n \times n}$, then $\left(\nabla u_{j}\right)$ converges in measure to $\nabla u$ in $U$ and $u$ is affine;

(3) the differential inclusion (4.2) is strongly rigid; i.e., if $\operatorname{dist}\left(\nabla u_{j}, \mathcal{K}\right) \rightarrow 0$ in measure in $U$ and $\left(u_{j}\right)$ converges to $u$ weakly* in $W^{1, \infty}\left(U, \mathbb{R}^{n}\right)$, then $\left(\nabla u_{j}\right)$ converges in measure to $\nabla u$ in $U$ and $u$ is affine;

(4) we have

$$
\mathcal{K}=\mathcal{K}^{\mathrm{qc}},
$$

where $\mathcal{K}^{\mathrm{qc}}$ denotes the quasiconvex envelope of $\mathcal{K}$; i.e.,

$$
\mathcal{K}^{\mathrm{qc}}:=\left\{\mathbb{A} \in \mathbb{M}^{n \times n}: f(\mathbb{A}) \leq \sup _{\mathcal{K}} f, \forall f: \mathbb{M}^{n \times n} \rightarrow \mathbb{R} \text { quasiconvex }\right\} .
$$


For the readers' convenience, the proof of Lemma 4.1 is included in Appendix A.

Below we give a list of nonempty compact sets $\mathcal{K} \subset \mathbb{M}^{n \times n}$ for which (4.1) holds true. The most prominent examples are due to Ball and James (1987, Proposition 2) and to Friesecke et al. (2002, Theorem 3.1) and correspond, respectively, to the case of two non rank-1 connected matrices and to that of $S O(n)$.

We notice that in the examples (1) and (3), property (4.1) directly follows from an incompatibility condition for the approximate solutions of (4.2), as shown in Chaudhuri and Müller (2004) (see also De Lellis and Székelyhidi 2006, Theorem 1.2). This condition reduces rigidity for multiple-wells to a single-well rigidity statement. We recall here that two disjoint compact sets $K_{1}, K_{2} \in \mathbb{M}^{n \times n}$ are incompatible for the differential inclusion (4.2), with $\mathcal{K}=K_{1} \cup K_{2}$, if for any sequence $\left(u_{j}\right) \subset W^{1, \infty}\left(U, \mathbb{R}^{n}\right)$ such that $\operatorname{dist}\left(\nabla u_{j}, K_{1} \cup K_{2}\right) \rightarrow 0$ in measure, then either $\operatorname{dist}\left(\nabla u_{j}, K_{1}\right) \rightarrow 0$ or $\operatorname{dist}\left(\nabla u_{j}, K_{2}\right) \rightarrow 0$ in measure. In this case, $K_{1}$ and $K_{2}$ are also called incompatible energy-wells.

In the examples (4) and (5) listed, property (4.1) is instead a consequence of the Friesecke, James, and Müller rigidity estimate (Friesecke et al. 2002, Theorem 3.1) for (2), and of the above mentioned incompatibility for approximate solutions of (4.2). Although equality (4.3) is a consequence of (4.1) as established by Lemma 4.1, for each example in the list below we also give a precise reference to a direct proof of (4.3). We refer the reader to Müller (1999), Kirchheim (2003) and Rindler (2018, Chapter 8) for more details on these topics.

(1) $\mathcal{K}=\left\{\mathbb{A}_{1}, \mathbb{A}_{2}\right\}$, where $\mathbb{A}_{1}, \mathbb{A}_{2} \in \mathbb{M}^{n \times n}$ are not rank-1 connected, see Ball and James (1987, Proposition 2), (see also Zhang 1992, Example 4.3);

(2) $\mathcal{K}=S O(n)$ (Friesecke et al. 2002, Theorem 3.1) (for (4.3) see Kinderlehrer (1988) and also Zhang (1992, Example 4.4));

(3) $\mathcal{K}=\left\{\mathbb{A}_{1}, \mathbb{A}_{2}, \mathbb{A}_{3}\right\}$, where $\mathbb{A}_{1}, \mathbb{A}_{2}, \mathbb{A}_{3} \in \mathbb{R}^{n \times n}$ are such that $\mathcal{K}$ has no rank-1 connections, see Šverák (1992, Section 4);

(4) $\mathcal{K}=\bigcup_{i=1}^{N} \mathbb{A}_{i} S O(2)$, where $\mathbb{A}_{i} \in \mathbb{R}^{2 \times 2}$ are such that $\operatorname{det} \mathbb{A}_{i}>0$ for all $i \in\{1,2,3\}$ and $\mathcal{K}$ has no rank-1 connections, see Šverák (1993, Theorem 2 and Remark 1);

(5) $\mathcal{K}=S O(3) \cup S O(3) \mathbb{H}$, where $\mathbb{H}=\operatorname{diag}\left(h_{1}, h_{2}, h_{3}\right), h_{1} \geq h_{2} \geq h_{3}>0$ and $h_{2} \neq 1$ (the latter condition is equivalent to $\mathcal{K}$ having no rank-1 connections). Additionally, one of the following two conditions must hold true:

(i) there exists $i$ such that $\left(h_{i}-1\right)\left(h_{i-1} h_{i+1}-1\right) \geq 0$ (here the indices are counted modulo 3);

(ii) $h_{1} \geq h_{2}>1>h_{3}>\frac{1}{3}$ or $3>h_{1}>1>h_{2} \geq h_{3}>0$;

see Dolzmann et al. (2000, Theorem 1.2).

We now recall an extension of the piecewise-rigidity result contained in Theorem 2.3 to the case of a compact set $\mathcal{K}$ for which (4.1) holds true. We state this result for $G S B V$-functions and we refer the reader to Chambolle et al. (2007, Theorem 2.1) for the original statement in the $S B V$-setting.

Theorem 4.2 Let $\mathcal{K} \subset \mathbb{M}^{n \times n}$ be a nonempty compact set for which (4.1) holds true and let $u \in(G S B V(\Omega))^{n}$ be such that $\mathcal{H}^{n-1}\left(J_{u}\right)<+\infty$ and $\nabla u \in \mathcal{K}$ a.e. in $\Omega$. 
Then, $u \in P R_{\mathcal{K}}(\Omega) ;$ i.e.,

$$
u(x)=\sum_{i \in \mathbb{N}}\left(\mathbb{A}_{i} x+b_{i}\right) \chi_{E_{i}}(x)
$$

with $\mathbb{A}_{i} \in \mathcal{K}$ for every $i \in \mathbb{N}, b_{i} \in \mathbb{R}^{n}$, and $\left(E_{i}\right)$ Caccioppoli partition of $\Omega$.

For $\varepsilon>0$, we consider the functionals $F_{\varepsilon}^{\mathcal{K}}: L^{1}\left(\Omega, \mathbb{R}^{n}\right) \times L^{1}(\Omega) \longrightarrow[0,+\infty]$ defined as

$$
F_{\varepsilon}^{\mathcal{K}}(u, v):= \begin{cases}\int_{\Omega}\left(k_{\varepsilon} \Phi(v) W(x, \nabla u)+\frac{V(v)}{\varepsilon}+\varepsilon|\nabla v|^{2}\right) \mathrm{d} x & (u, v) \in W^{1,2}\left(\Omega, \mathbb{R}^{n}\right) \times W^{1,2}(\Omega) \\ +\infty & 0 \leq v \leq 1 \text { a.e. in } \Omega \\ \text { otherwise }\end{cases}
$$

where $k_{\varepsilon} \rightarrow+\infty$, as $\varepsilon \rightarrow 0, W: \Omega \times \mathbb{M}^{n \times n} \rightarrow[0,+\infty)$ is a Borel function such that $W(x, \mathbb{A})=0$ for every $\mathbb{A} \in \mathcal{K}$. We assume moreover that for every $x \in \Omega$ and every $\mathbb{A} \in \mathbb{M}^{n \times n}$ it holds $W(x, \mathbb{A}) \geq \alpha \operatorname{dist}^{2}(\mathbb{A}, \mathcal{K})$, for some $\alpha>0$.

By combining Proposition 4.3 and Theorem 4.4, we can identify the $\Gamma$-limit of $F_{\varepsilon}^{\mathcal{K}}$. Now using Theorem 4.2 in place of Theorem 2.3 , these results can be proven by following exactly the same arguments employed in the proofs of Proposition 3.2 and Theorem 3.3, respectively.

The following proposition shows that the $\Gamma$-limit of $\left(F_{\varepsilon}^{\mathcal{K}}\right)$ (if it exists) is finite only on $P R_{\mathcal{K}}(\Omega)$.

Proposition 4.3 Let $\left(u_{\varepsilon}, v_{\varepsilon}\right) \subset W^{1,2}\left(\Omega, \mathbb{R}^{n}\right) \times W^{1,2}(\Omega), 0 \leq v_{\varepsilon} \leq 1$ a.e. in $\Omega$, be such that

$$
\liminf _{\varepsilon \rightarrow 0} F_{\varepsilon}^{\mathcal{K}}\left(u_{\varepsilon}, v_{\varepsilon}\right)<+\infty \text { and } u_{\varepsilon} \rightarrow u \text { in } L^{1}\left(\Omega, \mathbb{R}^{n}\right)
$$

Then, $v_{\varepsilon} \rightarrow 1$ in $L^{1}(\Omega)$ and $u \in P R_{\mathcal{K}}(\Omega)$.

In addition, the following $\Gamma$-convergence result holds true.

Theorem 4.4 The family $\left(F_{\varepsilon}^{\mathcal{K}}\right)$ defined in $(4.5) \Gamma\left(L^{1}\left(\Omega, \mathbb{R}^{n}\right) \times L^{1}(\Omega)\right)$-converges to the functional $F^{\mathcal{K}}: L^{1}\left(\Omega, \mathbb{R}^{n}\right) \times L^{1}(\Omega) \longrightarrow[0,+\infty]$ given by

$$
F^{\mathcal{K}}(u, v):= \begin{cases}2 C_{V} \mathcal{H}^{n-1}\left(J_{u}\right) & \text { if } u \in P R_{\mathcal{K}}(\Omega), v=1 \text { a.e. in } \Omega \\ +\infty & \text { otherwise }\end{cases}
$$

where $C_{V}:=2 \int_{0}^{1} \sqrt{V(s)} d s$.

\subsection{The Case of Linearised Elasticity}

An approximation result similar to that proven in Theorems 3.3 and 4.4 can be established for interfacial energies appearing in the context of linearised elasticity. In this 
case, the energy functionals are defined on piecewise-rigid displacements; i.e., on displacements of a linearly elastic body which does not store elastic energy.

Definition 4.5 A map $u: \Omega \rightarrow \mathbb{R}^{n}$ is called a piecewise-rigid displacement if there exist skew-symmetric matrices $\mathbb{A}_{i} \in \mathbb{M}_{\text {skew }}^{n \times n}$ and vectors $b_{i} \in \mathbb{R}^{n}$ such that

$$
u(x)=\sum_{i \in \mathbb{N}}\left(\mathbb{A}_{i} x+b_{i}\right) \chi_{E_{i}}(x),
$$

with $\left(E_{i}\right)$ Caccioppoli partition of $\Omega$. The set of piecewise-rigid displacements on $\Omega$ will be denoted by $P R D(\Omega)$.

The next piecewise-rigidity result corresponds to Theorems 2.3 and 4.2 in the setting of linearised elasticity. We state it here for $G S B D$ maps, the original version (Chambolle et al. 2007, Theorem A.1) being stated in the $S B D$-setting, though the method of proof works also in the more general $G S B D$-setting (see also Friedrich 2018, Theorem 2.1, Remark 2.2 ( $i$ ) for the two-dimensional case).

In what follows $e(u)$ denotes the symmetrised approximate gradient of $u \in$ $G S B D(\Omega)$ (cf. Dal Maso 2013).

Theorem 4.6 Let $u \in G S B D(\Omega)$ be such that $\mathcal{H}^{n-1}\left(J_{u}\right)<+\infty$ and $e(u)=0$ a.e. in $\Omega$. Then, $u \in P R D(\Omega)$.

To approximate interfacial energies defined on $P R D(\Omega)$, for $\varepsilon>0$, we consider the functionals $E_{\varepsilon}: L^{1}\left(\Omega, \mathbb{R}^{n}\right) \times L^{1}(\Omega) \longrightarrow[0,+\infty]$ defined as

$E_{\varepsilon}(u, v):= \begin{cases}\int_{\Omega}\left(k_{\varepsilon} \Phi(v) W(x, \nabla u) \mathrm{d} x+\frac{V(v)}{\varepsilon}+\varepsilon|\nabla v|^{2}\right) \mathrm{d} x & (u, v) \in W^{1,2}\left(\Omega, \mathbb{R}^{n}\right) \times W^{1,2}(\Omega), \\ +\infty & 0 \leq v \leq 1 \text { a.e. in } \Omega \\ & \text { otherwise, }\end{cases}$

where $k_{\varepsilon} \rightarrow+\infty$, as $\varepsilon \rightarrow 0, W: \Omega \times \mathbb{M}^{n \times n} \rightarrow[0,+\infty)$ is a Borel function such that $W(\cdot, \mathbb{A})=0$ if $\mathbb{A}$ is skew-symmetric. Moreover, for every $x \in \Omega$ and every $\mathbb{A} \in \mathbb{M}^{n \times n}$ it holds

$$
\alpha\left|\mathbb{A}^{\mathrm{sym}}\right|^{2} \leq W(x, \mathbb{A})
$$

for some $\alpha>0$. Here we denote by $\mathbb{A}^{\text {sym }}$ the symmetric part of $\mathbb{A}$, namely $\mathbb{A}^{\text {sym }}:=$ $\frac{\mathbb{A}+\mathbb{A}^{T}}{2}$. We use standard notation for the strain $e(u)=(\nabla u)^{\mathrm{sym}}$ of $u \in W^{1,2}\left(\Omega, \mathbb{R}^{n}\right)$.

Remark 4.7 We refer the reader to Conti et al. (2017, Remark 4.14) for an explicit example of a nonconvex, polyconvex function which depends non-trivially on the skew-symmetric part of $\mathbb{A}$ and satisfies the bounds

$$
\alpha\left|\mathbb{A}^{\mathrm{sym}}\right|^{2} \leq W(x, \mathbb{A}) \leq \beta\left(\left|\mathbb{A}^{\mathrm{sym}}\right|^{2}+1\right)
$$

for some $\alpha, \beta>0$, for every $x \in \Omega$ and for every $\mathbb{A} \in \mathbb{M}^{n \times n}$. 
Another example can be obtained by taking $W(\mathbb{A})=h^{2}(\mathbb{A}), \mathbb{A} \in \mathbb{M}^{n \times n}$, where $h$ is a one-homogeneous quasiconvex function such that for every $\mathbb{A} \in \mathbb{M}^{n \times n}$ and for some $\alpha, \beta>0$

$$
\alpha\left|\mathbb{A}^{\mathrm{sym}}\right| \leq h(\mathbb{A}) \leq \beta\left|\mathbb{A}^{\mathrm{sym}}\right|
$$

with $h$ depending non-trivially on $\mathbb{A}^{\text {skew }}:=\frac{\mathbb{A}-\mathbb{A}^{T}}{2}$. We notice that, in particular, $h$ (and therefore $W$ ) is not convex. A function as above can be obtained by slightly modifying Müller's celebrated example Müller (1992) similarly as in Caroccia et al. (2020, Section 7).

In the following proposition, we show that the $\Gamma$-limit of $\left(E_{\varepsilon}\right)$ (if it exists) is finite only on piecewise-rigid displacements.

Proposition 4.8 Let $\left(u_{\varepsilon}, v_{\varepsilon}\right) \subset W^{1,2}\left(\Omega, \mathbb{R}^{n}\right) \times W^{1,2}(\Omega)$ be such that

$$
\liminf _{\varepsilon \rightarrow 0} E_{\varepsilon}\left(u_{\varepsilon}, v_{\varepsilon}\right)<+\infty \text { and } u_{\varepsilon} \rightarrow u \text { in } L^{1}\left(\Omega, \mathbb{R}^{n}\right)
$$

Then, $v_{\varepsilon} \rightarrow 1$ in $L^{1}(\Omega)$ and $u \in P R D(\Omega)$.

Proof We argue by comparison as in the proof of Proposition 3.2 to infer that $v_{\varepsilon}$ converges to 1 strongly in $L^{1}(\Omega)$, and that the limit function $u$ satisfies

$$
e(u)=0 \text { a.e. in } \Omega, \text { and } \mathcal{H}^{n-1}\left(J_{u}\right)<+\infty
$$

now invoking (Chambolle and Crismale 2019, Theorem 5.1) (see also Iurlano 2014, Theorem 7) in place of Theorem 3.1. Eventually, the conclusion follows by Theorem 4.6.

Arguing as in the proof of Theorem 3.3, on account of Proposition 4.8 we can now prove the following $\Gamma$-convergence result for the family $\left(E_{\varepsilon}\right)$.

Theorem 4.9 The family of functionals $\left(E_{\varepsilon}\right)$ defined in $(4.8) \Gamma\left(L^{1}\left(\Omega, \mathbb{R}^{n}\right) \times L^{1}(\Omega)\right)$ converges to the functional $E: L^{1}\left(\Omega, \mathbb{R}^{n}\right) \times L^{1}(\Omega) \longrightarrow[0,+\infty]$ given by

$$
E(u, v):= \begin{cases}2 C_{V} \mathcal{H}^{n-1}\left(J_{u}\right) & \text { if } u \in P R D(\Omega) \text { and } v=1 \text { a.e. in } \Omega \\ +\infty & \text { otherwise }\end{cases}
$$

where $C_{V}:=2 \int_{0}^{1} \sqrt{V(s)} d s$.

Proof The lower bound inequality follows thanks to Chambolle and Crismale (2019, Theorem 5.1) (see also Iurlano 2014, Theorem 7).

The proof of the upper bound inequality in Step 2 of Theorem 3.3 remains unchanged up to replacing rotation matrices with skew-symmetric ones. 
Acknowledgements The work of M. Cicalese was supported by the DFG Collaborative Research Center TRR 109, "Discretization in Geometry and Dynamics". The work of C. I. Zeppieri was supported by the Deutsche Forschungsgemeinschaft (DFG, German Research Foundation) under the Germany Excellence Strategy EXC 2044-390685587, Mathematics Münster: Dynamics-Geometry-Structure. M. Focardi has been partially supported by GNAMPA.

Funding Open Access funding enabled and organized by Projekt DEAL.

Open Access This article is licensed under a Creative Commons Attribution 4.0 International License, which permits use, sharing, adaptation, distribution and reproduction in any medium or format, as long as you give appropriate credit to the original author(s) and the source, provide a link to the Creative Commons licence, and indicate if changes were made. The images or other third party material in this article are included in the article's Creative Commons licence, unless indicated otherwise in a credit line to the material. If material is not included in the article's Creative Commons licence and your intended use is not permitted by statutory regulation or exceeds the permitted use, you will need to obtain permission directly from the copyright holder. To view a copy of this licence, visit http://creativecommons.org/licenses/by/4.0/.

\section{Appendix A. Proof of the Rigidity Lemma}

Proof of Lemma 4.1 We start by showing statement (3), from which (1) and (2) immediately follow (we notice that actually the validity of (1) and (2) is equivalent to (3), as shown in Rindler (2018, Corollary 8.9). To this end let $\left(u_{j}\right)$ and $u$ be as in (3); then (dist $\left.\left(\nabla u_{j}, \mathcal{K}\right)\right)$ converges to 0 in $L^{p}(U)$, indeed it converges to 0 in measure and $\nabla u_{j}$ is bounded in $L^{\infty}\left(U, \mathbb{M}^{n \times n}\right)$. Thanks to (4.1) we can find $\mathbb{A}_{j} \in \mathcal{K}$ such that

$$
\left\|\nabla u_{j}-\mathbb{A}_{j}\right\|_{L^{p}\left(U, \mathbb{M}^{n \times n}\right)} \leq C\left\|\operatorname{dist}\left(\nabla u_{j}, \mathcal{K}\right)\right\|_{L^{p}(U)} .
$$

For an arbitrary subsequence $\left(j_{k}\right)$, we extract a further subsequence $\left(j_{k_{h}}\right)$ such that $\mathbb{A}_{j_{k}}$ converges to some $\mathbb{A} \in \mathcal{K}$. Therefore, $\left(\nabla u_{j_{k_{h}}}\right)$ converges to $\mathbb{A}$ in $L^{p}\left(U, \mathbb{M}^{n \times n}\right)$. This convergence combined with the weak ${ }^{*}$ convergence of $\left(u_{j}\right)$ to $u$ in $W^{1, \infty}\left(U, \mathbb{R}^{n}\right)$ immediately gives $\nabla u=\mathbb{A}$ a.e. on $U$. Being the limit independent of the subsequence, the Urysohn property implies that the whole sequence $\left(\nabla u_{j}\right)$ converges to $\mathbb{A}$ in $L^{p}\left(U, \mathbb{M}^{n \times n}\right)$ and hence the claim.

We finally prove (4) directly from (4.1), despite its validity is well-known in the literature as a consequence of (2) (cf. for instance (Müller 1999, Theorem 4.10). To conclude, we only need to prove that $\mathcal{K}^{\mathrm{qc}} \subseteq \mathcal{K}$. To do so, we use that

$$
\mathcal{K}^{\mathrm{qc}}=\left\{\mathbb{A} \in \mathbb{M}^{n \times n}: Q\left(\operatorname{dist}^{q}(\cdot, \mathcal{K})\right)(\mathbb{A})=0\right\}
$$

for every $q \in[1,+\infty$ ) (cf. Zhang 1992, Proposition 2.14, and also Müller 1999, Theorem 4.10). Let $\mathbb{A} \in \mathcal{K}^{\mathrm{qc}}$, then the definition of quasi-convex envelope of the distance function yields the existence of $\varphi_{j} \in W_{0}^{1, \infty}\left(U, \mathbb{R}^{n}\right)$ such that

$$
\lim _{j \rightarrow+\infty} \int_{U} \operatorname{dist}\left(\mathbb{A}+\nabla \varphi_{j}(x), \mathcal{K}\right) d x=0 .
$$


Moreover, the Zhang Lemma (cf. Zhang 1992, and also Müller 1999, Lemma 4.21) provides us with a sequence $\left(\phi_{j}\right) \subset W_{0}^{1, \infty}\left(U, \mathbb{R}^{n}\right)$ such that

$$
\sup _{j \in \mathbb{N}}\left\|\nabla \phi_{j}\right\|_{L^{\infty}\left(U, \mathbb{M}^{n \times n}\right)}<+\infty, \quad \lim _{j \rightarrow+\infty} \mathcal{L}^{n}\left(\left\{\phi_{j} \neq \varphi_{j}\right\}\right)=0 .
$$

Thus, being $\mathcal{K}$ compact and $\left(\nabla \phi_{j}\right)$ bounded in $L^{\infty}\left(U, \mathbb{M}^{n \times n}\right)$, we obtain

$$
\begin{aligned}
\int_{U} \operatorname{dist}^{p}\left(\mathbb{A}+\nabla \phi_{j}(x), \mathcal{K}\right) d x & \leq \int_{U} \operatorname{dist}^{p}\left(\mathbb{A}+\nabla \varphi_{j}(x), \mathcal{K}\right) d x+C \mathcal{L}^{n}\left(\left\{\phi_{j} \neq \varphi_{j}\right\}\right) \\
& \leq C \int_{U} \operatorname{dist}\left(\mathbb{A}+\nabla \varphi_{j}(x), \mathcal{K}\right) d x+C \mathcal{L}^{n}\left(\left\{\phi_{j} \neq \varphi_{j}\right\}\right),
\end{aligned}
$$

thus, eventually,

$$
\lim _{j \rightarrow+\infty} \int_{U} \operatorname{dist}^{p}\left(\mathbb{A}+\nabla \phi_{j}(x), \mathcal{K}\right) d x=0
$$

For $x \in U$ let $u_{j}(x)=\mathbb{A} x+\phi_{j}(x)$. By (4.1), there exists $\mathbb{A}_{j} \in \mathcal{K}$ such that

$$
\left\|\nabla u_{j}-\mathbb{A}_{j}\right\|_{L^{p}\left(U, \mathbb{M}^{n \times n}\right)} \leq C\left\|\operatorname{dist}\left(\nabla u_{j}, \mathcal{K}\right)\right\|_{L^{p}(U)},
$$

then by the Jensen inequality we get

$$
\limsup _{j \rightarrow+\infty} \mathcal{L}^{n}(U)\left|\mathbb{A}-\mathbb{A}_{j}\right|^{p} \leq \lim _{j \rightarrow+\infty} \int_{U}\left|\mathbb{A}-\mathbb{A}_{j}+\nabla \phi_{j}\right|^{p} d x=0,
$$

and therefore $\mathbb{A} \in \mathcal{K}$.

\section{References}

Alicandro, R., Braides, A., Shah, J.: Free-discontinuity problems via functionals involving the $L^{1}$-norm of the gradient and their approximations. Interfaces Free Bound. 1, 17-37 (1999)

Alicandro, R., Focardi, M.: Variational approximation of free-discontinuity energies with linear growth. Commun. Cont. Math. 4(4), 685-723 (2002)

Ambrosio, L.: The space $\operatorname{SBV}(\Omega)$ and free-discontinuity problems. In: Variational and Free Boundary Problems, IMA. Math. Appl., vol. 53, pp. 29-45. Springer, New York (1993)

Ambrosio, L., Fusco, N., Pallara, D.: Functions of Bounded Variation and Free Discontinuity Problems. Oxford University Press, New York (2000)

Ambrosio, L., Tortorelli, V.M.: Approximation of functionals depending on jumps by elliptic functionals via $\Gamma$-convergence. Commun. Pure Appl. Math. 43(8), 999-1036 (1990)

Ambrosio, L., Tortorelli, V.M.: On the approximation of free-discontinuity problems. Boll. Un. Mat. Ital. (7) 6-B, 105-123 (1992)

Bach, A., Braides, A., Zeppieri, C.I.: Quantitative analysis of finite-difference approximations of freediscontinuity functionals. Interfaces Free Bound. 22(3), 317-381 (2020)

Bach, A., Cicalese, M., Ruf, M.: Random finite-difference discretizations of the Ambrosio-Tortorelli functional with optimal mesh size. SIAM J. Math. Anal. 53(2), 2275-2318 (2021)

Bach, A., Marziani, R., Zeppieri, C.I.: Г-convergence and stochastic homogenisation of singularly-perturbed elliptic functionals. ArXiv preprint: arXiv:2102.09872 
Ball, J., James, R.: Fine phase mixtures as minimizers of energy. Arch. Ration. Mech. Anal. 100, 13-52 (1987)

Bourdin, B., Francfort, G.A., Marigo, J.-J.: Numerical experiments in revisited brittle fracture. J. Mech. Phys. Solids 48(4), 797-826 (2000)

Bourdin, B., Francfort, G.A., Marigo, J.-J.: The variational approach to fracture. J. Elast. 9, 5-148 (2008)

Braides, A.: Г-Convergence for Beginners. Oxford University Press, Oxford (2002)

Braides, A.: Approximation of Free Discontinuity Problems. Lecture Notes in Mathematics, vol. 1694. Springer, Berlin (1998)

Braides, A., Conti, S., Garroni, A.: Density of polyhedral partitions. Calc. Var. Partial Differ. Equ. 56(2), 1-10 (2017)

Caroccia, M., Van Goethem, N.: Damage-driven fracture with low-order potentials: asymptotic behavior, existence and applications. ESAIM Math. Model. Numer. Anal. 53(4), 1305-1350 (2019)

Caroccia, M., Focardi, M., Van Goethem, N.: On the integral representation of variational functionals on BD. SIAM J. Math. Anal. 52(4), 4022-4067 (2020)

Chambolle, A.: An approximation result for special functions with bounded deformation. J. Math. Pures Appl. 83, 929-954 (2004)

Chambolle, A., Crismale, V.: A density result in $G S B D^{p}$ with applications to the approximation of brittle fracture energies. Arch. Ration. Mech. Anal. 232(3), 1329-1378 (2019)

Chambolle, A., Giacomini, A., Ponsiglione, M.: Piecewise rigidity. J. Funct. Anal. 244, 134-153 (2007)

Chaudhuri, N., Müller, S.: Rigidity estimate for two incompatible wells. Calc. Var. Partial. Differ. Equ. 19, 379-390 (2004)

Clayton, J.D., Knap, J.: A geometrically nonlinear phase field theory of brittle fracture. Int. J. Fract. 189(2), 139-148 (2014)

Conti, S., Focardi, M., Iurlano, F.: Which special functions of bounded deformation have bounded variation? Proc. R. Soc. Edinb. Sect. A 148A, 33-50 (2017)

Conti, S., Focardi, M., Iurlano, F.: Phase field approximation of cohesive fracture models. Ann. I. H. Poincaré 33, 1033-1067 (2016)

Conti, S., Focardi, M., Iurlano, F.: Integral representation for functionals defined on $S B D^{p}$ in dimension two. Arch. Ration. Mech. Anal. 223(3), 1337-1374 (2017)

Crismale, V.: On the approximation of SBD functions and some applications. SIAM J. Math. Anal. 51(6), 5011-5048 (2019)

Dal Maso, G.: An Introduction to $\Gamma$-Convergence, vol. 8. Springer, Berlin (2012)

Dal Maso, G.: Generalised functions of bounded deformation. J. Eur. Math. Soc. 15(5), 1943-1997 (2013)

Dal Maso, G., Francfort, G., Toader, R.: Quasistatic crack growth in nonlinear elasticity. Arch. Ration. Mech. Anal. 176(2), 165-225 (2005)

Dal Maso, G., Iurlano, F.: Fracture models as $\Gamma$-limits of damage models. Commun. Pure Appl. Anal. 12, $1657-1686$ (2013)

De Giorgi, E., Ambrosio, L.: New functionals in the calculus of variations. Atti Accad. Naz. Lincei Rend. Cl. Sci. Fis. Mat. Natur. 82, 199-210 (1988)

De Lellis, C., Székelyhidi, L.: Simple proof of two-well rigidity. C. R. Math. Acad. Sci. Paris 343(5), 367-370 (2006)

Dolzmann, G., Kirchheim, B., Müller, S., Šverák, V.: The two-well problem in three dimensions. Calc. Var. Partial Differ. Equ. 10(1), 21-40 (2000)

Focardi, M.: On the variational approximation of free-discontinuity problems in the vectorial case. Math. Models Methods Appl. Sci. 11(4), 663-684 (2001)

Focardi, M.: Variational Approximation of Vectorial Free Discontinuity Problems: The Discrete and Continuous Case. PhD thesis, Scuola Normale Superiore, Pisa (2002)

Focardi, M., Iurlano, F.: Asymptotic analysis of Ambrosio-Tortorelli energies in linearized elasticity. SIAM J. Math. Anal. 46, 2936-2955 (2014)

Francfort, G., Marigo, J.-J.: Revisiting brittle fracture as an energy minimization problem. J. Mech. Phys. Solids 46, 1319-1342 (1998)

Friedrich, M.: A piecewise Korn inequality in SBD and applications to embedding and density results. SIAM J. Math. Anal. 50, 3842-3918 (2018)

Friedrich, M., Solombrino, F.: Functionals defined on piecewise rigid functions: integral representation and $\Gamma$-convergence. Arch. Ration. Mech. Anal. 236, 1325-1387 (2020)

Friesecke, G., James, R., Müller, S.: A theorem on geometric rigidity and the derivation of nonlinear plate theory from three-dimensional elasticity. Commun. Pure Appl. Math. 55(11), 1461-1506 (2002) 
Giusti, E.: Direct Methods in the Calculus of Variations. World Scientific Publishing Co., River Edge (2003)

Griffith, A.A.: The phenomena of rupture and flow in solids. Philos. Trans. R. Soc. Lond. 221, 163-198 (1920)

Iurlano, F.: Fracture and plastic models as $\Gamma$-limits of damage models under different regimes. Adv. Calc. Var. 6, 165-189 (2013)

Iurlano, F.: A density result for $G S B D$ and its application to the approximation of brittle fracture energies. Calc. Var. Partial Differ. Equ. 51(1-2), 315-342 (2014)

Kinderlehrer, D.: Remarks about equilibrium configurations of crystals. In: Material instabilities in Continuum Mechanics (Edinburgh, 1985-1986), pp. 217-241. Oxford Sci. Publ., Oxford Univ. Press, New York (1988)

Kirchheim, B.: Rigidity and Geometry of Microstructures. Lecture notes 16, Max-Planck-Institut für Mathematik in den Naturwissenschaften, Leipzig (2003)

Kristensen, J.: Lower semicontinuity in spaces of weakly differentiable functions. Math. Ann. 313(4), 653-710 (1999)

Modica, L.: The gradient theory of phase transitions and the minimal interface criterion. Arch. Ration. Mech. Anal. 98, 123-142 (1987)

Modica, L., Mortola, S.: Un esempio di Г-convergenza. Boll. Un. Mat. Ital. 14-B, 285-299 (1977)

Müller, S.: On quasiconvex functions which are homogeneous of degree 1. Indiana Univ. Math. J. 41(1), 295-301 (1992)

Müller, S.: Variational models for microstructure and phase transitions. In: Calculus of Variations and Geometric Evolution Problems (Cetraro, 1996). Lecture Notes in Mathematics, vol. 1713, pp. 85210. Springer, Berlin (1999)

Mumford, D., Shah, J.: Optimal approximations by piecewise smooth functions and associated variational problems. Commun. Pure Appl. Math. 42(5), 577-685 (1989)

Pham, K., Marigo, J.-J.: Approche variationnelle de l'endommagement: I. Les concepts fondamentaux. Comptes Rendus Mécanique 338, 191-198 (2010)

Pham, K., Marigo, J.-J.: Approche variationnelle de l'endommagement: II. Les modèles à gradient. Comptes Rendus Mécanique 338, 199-206 (2010)

Pham, K., Amor, H., Marigo, J.-J., Maurini, C.: Gradient damage models and their use to approximate brittle fracture. Int. J. Damage Mech. 20(4), 618-652 (2011)

Rindler, F.: Calculus of Variations. Universitext. Springer, Cham (2018)

Šverák, V.: New examples of quasiconvex functions. Arch. Ration. Mech. Anal. 119(4), 293-300 (1992)

Šverák, V.: On the problem of two wells. In: Microstructure and Phase Transition, IMA Math. Appl., vol. 54, pp. 183-189. Springer, New York (1993)

Vicente, D.: An anisotropic Mumford Shah model. J. Math. Anal. Appl. 447, 181-205 (2017)

Wu, J.-Y., Nguyen, V.P., Nguyen, C.T., Sutula, D., Bordas, S., Sinaie, S.: Phase field modeling of fracture. In: Advances in Applied Mechancis: Multi-Scale Theory and Computation, vol. 52 (2018)

Zhang, K.: A construction of quasiconvex functions with linear growth at infinity. Ann. Scuola Norm. Sup. Pisa Cl. Sci. (4) 19(3), 313-326 (1992)

Zhang, K.: Quasiconvex functions, $S O(n)$ and two elastic wells. Ann. Inst. H. Poincarè Anal. Non Linèaire 14(6), 759-785 (1997)

Publisher's Note Springer Nature remains neutral with regard to jurisdictional claims in published maps and institutional affiliations. 